\title{
A REARRANGED GOOD $\lambda$ INEQUALITY \\ BY
}

RICHARD J. BAGBY AND DOUGLAS S. KURTZ

Abstract. Let $T f$ be a maximal Calderón-Zygmund singular integral, $M f$ the Hardy-Littlewood maximal function, and $w$ an $A_{\infty}$ weight. We replace the "good $\lambda$ " inequality

$$
w(\{x: T f(x)>2 \lambda \text { and } M f(x) \leqslant \varepsilon \lambda\}) \leqslant C(\varepsilon) w(\{x: T f(x)>\lambda\})
$$

by the rearrangement inequality

$$
(T f)_{w}^{*}(t) \leqslant C(M f)_{w}^{*}(t / 2)+(T f)_{w}^{*}(2 t)
$$

and show that it gives better estimates for $T f$. In particular, we obtain best possible weighted $L^{p}$ bounds, previously unknown exponential integrability estimates, and simplified derivations of known unweighted estimates for $(T f)^{*}$.

1. Introduction. In 1974, R. R. Coifman and C. Fefferman [6] published an elegant proof that Calderón-Zygmund singular integral operators are continuous in $L^{p}\left(\mathbf{R}^{n}, d \mu\right)$, provided that $d \mu=w(x) d x$, with $w$ in Muckenhoupt's weight class $A_{p}$. The heart of their proof is the inequality

$$
\mu\left(\left\{x \in \mathbf{R}^{n}: T f(x)>2 \lambda \text { and } M f(x) \leqslant \varepsilon \lambda\right\}\right) \leqslant C(\varepsilon) \mu\left(\left\{x \in \mathbf{R}^{n}: T f(x)>\lambda\right\}\right)
$$

for the maximal singular integral operator $T f$ and the Hardy-Littlewood maximal function $M f$ (see $\$ 2$ for the relevant definitions). The constant $C(\varepsilon) \rightarrow 0$ as $\varepsilon \rightarrow 0$. Such an inequality is called a "good $\lambda$ " inequality.

While useful estimates for operators are often given as functional inequalities for distribution functions as above, it is sometimes helpful to reformulate the estimates in terms of rearrangements. Since the rearrangement

$$
f^{*}(t)=\inf \left\{\lambda>0: \mu\left(\left\{x \in \mathbf{R}^{n}:|f(x)|>\lambda\right\}\right) \leqslant t\right\}
$$

satisfies $\mu\left(\left\{x \in \mathbf{R}^{n}:|f(x)|>\lambda\right\}\right)=\sup \left\{t>0: f^{*}(t)>\lambda\right\}$, such calculations are theoretically equivalent. In practice, neither the distribution function of $T f$ nor the rearrangement $(T f)^{*}$ is known exactly and the estimates for the latter may be superior.

Here we derive a rearrangement analog of the "good $\lambda$ " inequality for maximal singular integral operators in $\mathbf{R}^{n}$ :

$$
(T f)_{w}^{*}(t) \leqslant C(M f)_{w}^{*}(t / 2)+(T f)_{w}^{*}(2 t) .
$$

Received by the editors February 3, 1984.

1980 Mathematics Subject Classification. Primary 42B20, 42B25, 47B38.

Key words and phrases. Singular integral operator, maximal function, weight, rearrangement. 
Inequalities of this form have appeared previously in the work of Bennett and Sharpley [4]. With regard to our work, several points should be emphasized. First, our inequality (1.1) is not derived from the "good $\lambda$ " inequality; it is obtained as a direct estimate. Both inequalities are proved using similar techniques; in some ways our proof is simpler. In both cases, the unweighted and weighted results are consequences of a local estimate using Lebesgue measure. Second, our inequality is better than the "good $\lambda$ " inequality in that it is easier to use in many applications and seems to contain more information.

The superiority of our rearrangement inequality is due to the absence of the parameter $\varepsilon$ appearing in the "good $\lambda$ " inequality. To derive $L^{p}$ results from the "good $\lambda$ " inequality, one must choose $\varepsilon$ small enough that $C(\varepsilon)<2^{-p}$. This is inappropriate for estimates corresponding to $p=\infty$ (such as exponential integrability), while our rearrangement inequality easily gives the best possible results in these cases.

We obtain some interesting new results by iterating our basic inequality (1.1). Our main result is

THEOREM 1. Let Tf be a maximal singular integral defined by a Calderón-Zygmund kernel and $w \in A_{\infty}$. There is a $C>0$ such that

$$
(T f)_{w}^{*}(t) \leqslant C(M f)_{w}^{*}\left(\frac{t}{2}\right)+C \int_{t}^{\infty}(M f)_{w}^{*}(s) \frac{d s}{s}
$$

for all $t>0$.

When $w \equiv 1$, this reduces to

$$
(T f)^{*}(t) \leqslant C\left\{\frac{1}{t} \int_{0}^{t} f^{*}(s) d s+\int_{t}^{\infty} f^{*}(s) \frac{d s}{s}\right\},
$$

proved by Bennett and Rudnick [3]. A second consequence of (1.1) is

THEOREM 2. Let $T f$ be a maximal singular integral defined by a Calderón-Zygmund kernel and $w \in A_{\infty}$. There is a constant $C>0$ so that

$$
(T f)_{w}^{* *}(t) \leqslant(T f)_{w}^{*}(t)+C(M f)_{w}^{*}(t)
$$

for all $t>0$.

This result has several interesting consequences. Bennett, De Vore and Sharpley $[1,2]$ proved that $T$ is bounded from $L^{\infty}$ to BMO and that unweighted rearrangements satisfy $f^{* *}(t) \leqslant f^{*}(t)+C\|f\|_{\mathrm{BMO}}$; combining these results in the unweighted case gives our conclusion with $(M f)^{*}(t)$ replaced by $\|f\|_{\infty}$.

The paper is divided into five sections. In $\$ 2$ we consider definitions and background results. The main theorems are proved in $\$ 3$, including the proof of (1.1). Applications of these results and generalizations to Dini kernels are considered in the last two sections.

In the following we always assume that $\mu$ is a regular Borel measure on $\mathbf{R}^{n}$, generally of the form $d \mu=w(x) d x$. All sets and functions are measurable with respect to $\mu$.

We would like to thank Colin Bennett for some helpful discussions. 
2. Preliminary results. Let $\mu$ be a measure and $g$ be a function measurable with respect to $\mu$. Define the nonincreasing rearrangement of $g$ with respect to $\mu$ by

$$
g^{*}(t)=g_{\mu}^{*}(t)=\inf \{\lambda>0: \mu(\{x:|g(x)|>\lambda\}) \leqslant t\} .
$$

The averaged rearrangement of $g$ is

$$
g^{* *}(t)=\frac{1}{t} \int_{0}^{t} g^{*}(s) d s=\sup _{\mu(E) \leqslant t} \frac{1}{t} \int_{E}|g(x)| d \mu,
$$

where the supremum is taken over all $\mu$-measurable sets $E$ of measure at most $t$.

In the case that $\mu$ is Lebesgue measure, we use $|E|$ for the Lebesgue measure of $E$. If $\mu$ is an absolutely continuous measure (with respect to Lebesgue measure) given by integration of a function $w$, we use $w(E)$ to stand for $\int_{E} w(x) d x$.

In the proof of the main theorem, we will need the following version of Fatou's Lemma. The proof is included for completeness.

LEMMA 2.1. Let $\mu$ be a regular Borel measure on $\mathbf{R}^{n}$ and suppose $f, f_{n}, n=1,2, \ldots$, are $\mu$-measurable functions such that $f_{n}$ converges to $f$ uniformly on compact subsets of $\mathbf{R}^{n}$. If $f_{n}^{*}(t) \leqslant G(t), 0<t<\infty$, then $f^{*}(t) \leqslant G(t), 0<t<\infty$.

Proof. Fix $t>0$ and let $\lambda>G(t)$. Given a compact set, $K$, choose $n$ such that $\left|f(x)-f_{n}(x)\right|<\lambda-G(t)$ for all $x \in K$. This implies that $|f(x)| \leqslant\left|f_{n}(x)\right|+\lambda-$ $G(t)$ on $K$ so that

$$
\begin{aligned}
\mu(\{x \in K:|f(x)|>\lambda\}) & \leqslant \mu\left(\left\{x \in K:\left|f_{n}(x)\right|>G(t)\right\}\right) \\
& \leqslant \mu\left(\left\{x \in K:\left|f_{n}(x)\right|>f_{n}^{*}(t)\right\}\right) \leqslant t .
\end{aligned}
$$

By regularity, $\mu\left(\left\{x \in \mathbf{R}^{n}:|f(x)|>\lambda\right\}\right) \leqslant t$ for all $\lambda>G(t)$, so that $f^{*}(t) \leqslant G(t)$.

Consider a Calderón-Zygmund kernel $K(x), x \in \mathbf{R}^{n}$, satisfying the conditions:

(i) $|K(x)| \leqslant C /|x|^{n}$,

$$
\begin{aligned}
& \text { (ii) } \int_{\{\alpha<|x|<\beta\}} K(x) d x=0, \quad 0<\alpha<\beta, \\
& \text { (iii) }|K(x-y)-K(x)| \leqslant C|y| /|x|^{n+1} \text { for } 2|y| \leqslant|x| .
\end{aligned}
$$

Set $T_{\varepsilon} f(x)=\int_{\{y:|x-y|>\varepsilon\}} K(x-y) f(y) d y$. We define the singular integral associated to $K$ as

$$
K * f=\lim _{\varepsilon \downarrow 0} T_{\varepsilon} f
$$

and the maximal singular integral by

$$
T f=\sup _{\varepsilon>0}\left|T_{\varepsilon} f\right| .
$$


We will be using the Hardy-Littlewood maximal function to control singular integrals. This operator is defined by

$$
M f(x)=\sup \frac{1}{|Q|} \int_{Q}|f(y)| d y,
$$

where the supremum is taken over all cubes containing $x$.

Since $M F \equiv \infty$ unless $f$ is locally integrable and all of our results give upper bounds in terms of $M f$, we may assume $f$ is locally integrable throughout. Still, our assumptions on $f$ do not guarantee the absolute convergence of the integral defining $T_{\varepsilon} f(x)$. We interpret

$$
T_{\varepsilon} f(x)=\lim _{R \rightarrow \infty} \int_{\{\varepsilon<|x-y| \leqslant R\}} f(y) K(x-y) d y
$$

whenever this limit exists and $\left|T_{\varepsilon} f(x)\right|=\infty$ when it does not; our estimates are then both meaningful and correct.

We say $\mu$ is a doubling measure if there is a constant $C=C(\mu)$ so that whenever $Q$ is a cube in $\mathbf{R}^{n}$ and $2 Q$ is the cube concentric with $Q$ having twice the side length, then $\mu(2 Q) \leqslant C \mu(Q)$. The conclusion of the following lemma is a standard condition for the almost everywhere existence of singular integrals (see, e.g., [3] for a discussion of these ideas).

LEMMA 2.2. Let $\mu$ be a doubling measure. If $\int_{a}^{\infty}(M f)_{\mu}^{*}(s) d s / s<\infty$ for some $a>0$, then $\int_{\mathbf{R}^{n}}|f(x)|(1+|x|)^{-n} d x<\infty$.

ProOF. First note that we can construct a sequence of disjoint cubes $\left\{Q_{k}\right\}$ with $Q_{1} \subset 2 Q_{k}$ for all $k$, so that $\mu\left(Q_{k}\right) \geqslant C>0$. Consequently, by picking a cube which contains sufficiently many of these cubes $Q_{k}$ we can choose a cube $Q$ centered at the origin for which $\mu(Q) \geqslant 2 a$. We then have

$$
\begin{aligned}
\int_{Q}|f(x)|(1+|x|)^{-n} d x & \leqslant \int_{Q}|f(x)| d x \leqslant|Q| \inf \{M f(x): x \in Q\} \\
& \leqslant|Q|(M f)_{\mu}^{*}(2 a) \leqslant C|Q| \int_{a}^{2 a}(M f)_{\mu}^{*}(t) \frac{d t}{t} .
\end{aligned}
$$

Let $t_{k}=\mu\left(2^{k} Q\right), I(k)=\left[t_{k-1}, t_{k}\right]$, and $E(k)=2^{k} Q \sim 2^{k-1} Q, k=1,2, \ldots$ Since $2^{k} Q$ is covered by the triples of (the maximal) disjoint subcubes of $E(k)$,

$$
t_{k} \leqslant C_{0} \mu(E(k))=C_{0}\left[t_{k}-t_{k-1}\right] \text {. }
$$

Consequently,

$$
\begin{aligned}
\int_{E(k)}|f(x)|(1+|x|)^{-n} d x & \leqslant C_{n}\left|2^{k} Q\right|^{-1} \int_{2^{k} Q}|f(x)| d x \\
& \leqslant C_{n} \inf \left\{M f(x): x \in 2^{k} Q\right\} \leqslant C_{n}(M f)_{\mu}^{*}\left(t_{k}\right) \\
& \leqslant C_{n} C_{0}(M f)_{\mu}^{*}\left(t_{k}\right)\left[t_{k}-t_{k-1}\right] / t_{k} \\
& \leqslant C_{n} C_{0} \int_{I(k)}(M f)_{\mu}^{*}(t) \frac{d t}{t}
\end{aligned}
$$


Therefore,

$$
\begin{aligned}
\int_{\mathbf{R}^{n}} \mid & f(x) \mid(1+|x|)^{-n} d x \\
& =\int_{Q}|f(x)|(1+|x|)^{-n} d x+\sum_{k=1}^{\infty} \int_{E(k)}|f(x)|(1+|x|)^{-n} d x \\
& \leqslant C|Q| \int_{a}^{2 a}(M f)_{\mu}^{*}(t) \frac{d t}{t}+C_{n} C_{0} \sum_{k=1}^{\infty} \int_{I(k)}(M f)_{\mu}^{*}(t) \frac{d t}{t} \\
& \leqslant C(|Q|+1) \int_{a}^{\infty}(M f)_{\mu}^{*}(s) \frac{d s}{s},
\end{aligned}
$$

so that the finiteness of the integral involving $M f$ implies the finiteness of the integral involving $f$.

In general we will consider weights $w$ that satisfy the $A_{\infty}$ condition of Muckenhoupt. We say that $w$ is in $A_{\infty}$ if there are constants $C, p \geqslant 1$ so that given any cube, $Q$, and any measurable subset $E \subset Q$,

$$
\frac{w(Q)}{w(E)} \leqslant C\left(\frac{|Q|}{|E|}\right)^{p} .
$$

Notice that such a $w$ defines a doubling measure. Coifman and Fefferman [6] prove that such a measure satisfies the following uniform absolute continuity condition: Given an $\varepsilon>0$, there is a $\delta>0$ such that for any cube $Q$ and measurable $E \subset Q$, $|E|<\delta|Q|$ implies $w(E)<\varepsilon w(Q)$.

3. Main results. In this section we prove Theorems 1 and 2 . The ideas needed to prove our first lemma are the same as those used by Coifman and Fefferman [6], adapted to rearrangements. In the following lemma, we only assume our functions are measurable. Thus we do not assert that a set of the form $E=\left\{x \in \mathbf{R}^{n}\right.$ : $T f(x)>\lambda\}$ is open.

LEMMA 3.1. Let $T f=\sup _{\varepsilon}>0\left|T_{\varepsilon} f\right|$ be a maximal singular integral operator with a kernel, $K$, satisfying the conditions of (2.1), and let $w \in A_{\infty}$. For all $\gamma, 0<\gamma<1$, there exists a constant $C=C(\gamma)>0$ such that

$$
(T F)_{w}^{*}(t) \leqslant C(M f)_{w}^{*}(\gamma t)+(T f)_{w}^{*}(2 t) \quad \text { for all } t>0 .
$$

Proof. Fix $t>0$ and set $E=E(2 t)=\left\{x \in \mathbf{R}^{n}\right.$ : Tf $\left.(x)>(T f)_{w}^{*}(2 t)\right\}$. Then $E$ is contained in an open set $\Omega$ with $w(\Omega) \leqslant 3 t$. By the Whitney decomposition theorem [10, p. 16], $\Omega=\cup Q_{k}$, with $\sum_{k=1}^{\infty} w\left(Q_{k}\right)=w(\Omega) \leqslant 3 t$. The $Q_{k}$ 's are disjoint cubes that satisfy

$$
\operatorname{diameter}\left(Q_{k}\right) \leqslant \operatorname{distance}\left(Q_{k}, \mathbf{R}^{n}-\Omega\right) \leqslant 4 \operatorname{diameter}\left(Q_{k}\right) .
$$

It is well known (see [10], for example) that there is a constant $A>0$, independent of $f$ and $\lambda>0$, such that

$$
\left|\left\{x \in \mathbf{R}^{n}: T f(x)>\lambda\right\}\right| \leqslant \frac{A}{\lambda} \int|f(x)| d x .
$$


Using this inequality, we show that for arbitrary $\delta>0$, there is a constant $C$ depending only on $A, n$, and $\delta$ for which

$$
\left|\left\{x \in Q_{k}: T f(x)>C M f(x)+(T f)_{w}^{*}(2 t)\right\}\right| \leqslant \delta\left|Q_{k}\right| \text {. }
$$

Choose $\delta$ small enough that $E \subset Q$ and $|E|<\delta|Q|$ implies $w(E)<(1-\gamma) w(Q) / 3$; then summing over all cubes $Q_{k}$ yields

$$
w\left(\left\{x \in \mathbf{R}^{n}: T f(x)>C M f(x)+(T f)_{w}^{*}(2 t)\right\}\right) \leqslant(1-\gamma) t .
$$

Since $w\left(\left\{x \in \mathbf{R}^{n}: M f(x)>(M f)_{w}^{*}(\gamma t)\right\}\right) \leqslant \gamma t$, it follows that

$$
\begin{aligned}
& w\left(\left\{x \in \mathbf{R}^{n}: T f(x)>C(M f)_{w}^{*}(\gamma t)+(T f)_{w}^{*}(2 t)\right\}\right) \\
& \leqslant w\left(\left\{x \in \mathbf{R}^{n}: T f(x)>C M f(x)+(T f)_{w}^{*}(2 t)\right\}\right) \\
&+w\left(\left\{x \in \mathbf{R}^{n}: C M f(x)>C(M f)_{w}^{*}(\gamma t)\right\}\right) \leqslant t .
\end{aligned}
$$

The definition of rearrangements then gives

$$
(T f)_{w}^{*}(t) \leqslant C(M f)_{w}^{*}(\gamma t)+(T f)_{w}^{*}(2 t) .
$$

In order to prove (3.1), fix $k$, choose $x_{k} \in \mathbf{R}^{n}-\Omega$ so that distance $\left(x_{k}, Q_{k}\right) \leqslant$ 4 diameter $\left(Q_{k}\right)$, and let $Q$ be the cube centered at $x_{k}$ having diameter equal to twenty times the diameter of $Q_{k}$. Next, write $f=g+h=f \chi_{Q}+f \chi_{F}$, where $F=\mathbf{R}^{n}-Q$. We shall show

$$
T h(x) \leqslant C_{1} M f(x)+(T f)_{w}^{*}(2 t), \quad x \in Q_{k},
$$

and

$$
\left|\left\{x \in Q_{k}: \operatorname{Tg}(x)>C_{2} M f(x)\right\}\right| \leqslant \delta\left|Q_{k}\right| ;
$$

setting $C=C_{1}+C_{2}$ then gives (3.1).

The second inequality follows by choosing $\lambda=\left(C_{2} /|Q|\right) \int|g(x)| d x$ and noting that $C_{2} M f(x) \geqslant \lambda$ on $Q_{k}$. Consequently,

$$
\left|\left\{x \in Q_{k}: \operatorname{Tg}(x)>C_{2} M f(x)\right\}\right| \leqslant|\{x: \operatorname{Tg}(x)>\lambda\}| \leqslant \frac{A}{\lambda} \int|g(x)| d x \leqslant \frac{A|Q|}{C_{2}} \text {. }
$$

By choosing $C_{2}$ sufficiently large, $A|Q| / C_{2} \leqslant \delta\left|Q_{k}\right|$.

For the first inequality, we prove for all $\varepsilon>0$ and $x \in Q_{k}$ that

$$
\left|T_{\varepsilon} h(x)\right| \leqslant T f\left(x_{k}\right)+C_{1} M f(x) .
$$

Since $T f\left(x_{k}\right) \leqslant(T f)_{w}^{*}(2 t)$, this would prove (3.1).

Fix $x \in Q_{k}, \varepsilon>0$, and set $r=\max \left[\varepsilon\right.$, distance $\left.\left(x_{k}, F\right)\right]$; note that

$$
r>10 \text { diameter }\left(Q_{k}\right) \text {. }
$$

Let $\Delta$ be the symmetric difference of the two balls $B(x, \varepsilon)$ and $B\left(x_{k}, \varepsilon\right)$. Then,

$$
\begin{aligned}
\left|T_{\varepsilon} h(x)\right| & =\left|\int_{\{y:|x-y|>\varepsilon\}} K(x-y) h(y) d y\right| \\
& \leqslant\left|\int_{\left\{y:\left|x_{k}-y\right|>\varepsilon\right\}} K(x-y) h(y) d y\right|+\int_{\Delta}|K(x-y) h(y)| d y=I_{1}+I_{2} .
\end{aligned}
$$


Note that $\Delta \subset B(x, 3 r)$ and that $t \in \operatorname{supp}(h)$ implies that $|x-t|>r / 2$. Thus, using condition (2.1)(i), it follows that $I_{2} \leqslant C_{3} M f(x)$. To estimate $I_{1}$, observe that it is bounded by

$$
\begin{aligned}
& \left|\int_{\left\{y:\left|x_{k}-y\right|>r\right\}} K\left(x_{k}-y\right) f(y) d y\right| \\
& \quad+\int_{\left\{y:\left|x_{k}-y\right|>r\right\}}\left|K\left(x_{k}-y\right)-K(x-y)\right||f(y)| d y .
\end{aligned}
$$

By condition (2.1)(iii),

$$
\begin{aligned}
\left|K\left(x_{k}-y\right)-K(x-y)\right| & \leqslant C\left|x_{k}-x\right| /|x-y|^{n+1} \\
& \leqslant C \operatorname{diameter}\left(Q_{k}\right) /|x-y|^{n+1} .
\end{aligned}
$$

Thus, with $d=\operatorname{diameter}\left(Q_{k}\right)$,

$$
\begin{aligned}
I_{1} & \leqslant T f\left(x_{k}\right)+C \int_{\{y:|x-y|>d\}}|f(y)|\left[d /|x-y|^{n+1}\right] d y \\
& \leqslant T f\left(x_{k}\right)+C_{4} M f(x) .
\end{aligned}
$$

Thus $T_{\varepsilon} h(x) \leqslant T f\left(x_{k}\right)+C_{1} M f(x)$, with $C_{1}=C_{3}+C_{4}$.

Unlike the parameter $\varepsilon$ in the "good $\lambda$ " inequalities, the constant $\gamma$ need not be varied. In all our applications we can simply take $\gamma=1 / 2$, as in (1.1). Furthermore, in the case of Lebesgue measure, we could replace $(M f)^{*}(t / 2)$ by $(M f)^{*}(t)$, due to the equivalence of $(M f)^{*}$ and $f^{* *}$.

LeMmA 3.2. Let $T f=\sup _{\varepsilon>0}\left|T_{\varepsilon} f\right|$ be a maximal singular integral operator with a kernel satisfying the conditions of (2.1), and let $w \in A_{\infty}$. There exists a constant $C>0$ such that

$$
(T f)_{w}^{*}(t) \leqslant C(M f)_{w}^{*}\left(\frac{t}{2}\right)+C \int_{t}^{\infty}(M f)_{w}^{*}(s) \frac{d s}{s}
$$

for all $t>0$ and functions $f$ satisfying $\lim _{t \rightarrow \infty}(T f)_{w}^{*}(t)=0$.

Proof. By iterating Lemma 3.1 with $\gamma=1 / 2$, we have

$$
(T f)_{w}^{*}(t) \leqslant C \sum_{k=0}^{\infty}(M f)_{w}^{*}\left(2^{k-1} t\right)+\lim _{t \rightarrow \infty}(T f)_{w}^{*}(t) .
$$

Therefore,

$$
(T f)_{w}^{*}(t) \leqslant 2 C(M f)_{w}^{*}(t / 2)+C \sum_{k=2}^{\infty}(M f)_{w}^{*}\left(2^{k-1} t\right) .
$$

Since

$$
(M f)_{w}^{*}\left(2^{k-1} t\right) \leqslant \int_{\left\{s: 2^{k-2} t \leqslant s \leqslant 2^{k-1} t\right\}}(M f)_{w}^{*}(s) \frac{d s}{s},
$$

the proof of the lemma is completed.

Theorems 1 and 2 now follow easily. 
Proof of Theorem 1. Fix $t>0$. Without loss of generality, assume $\int_{t}^{\infty}(M f)_{w}^{*}(s) d s / s$ is finite. For $k=1,2, \ldots$, write

$$
f=f_{k, 1}+f_{k, 2}=f(x) \chi(\{x:|x| \leqslant k\})+f(x) \chi(\{x:|x|>k\}) .
$$

By Lemma 2.2, with $d \mu=w(x) d x, T f_{k, 2}$ converges uniformly to 0 on all compact sets which implies that $T f_{k, 1}$ converges uniformly to $T f$ on compact sets. Since each $f_{k, 1}$ has compact support, $T f_{k, 1}(x) \rightarrow 0$ as $|x| \rightarrow \infty$ for all $k$. Therefore, we have that $\lim _{t \rightarrow \infty}\left(T f_{k, 1}\right)_{w}^{*}(t)=0$ for each $k$, so that by Lemma 3.2,

$$
\begin{aligned}
\left(T f_{k, 1}\right)_{w}^{*}(t) & \leqslant C\left(M f_{k, 1}\right)_{w}^{*}\left(\frac{t}{2}\right)+C \int_{t}^{\infty}\left(M f_{k, 1}\right)_{w}^{*}(s) \frac{d s}{s} \\
& \leqslant C(M f)_{w}^{*}\left(\frac{t}{2}\right)+C \int_{t}^{\infty}(M f)_{w}^{*}(s) \frac{d s}{s}
\end{aligned}
$$

Applying Lemma 2.1 with

$$
G(t)=C(M f)_{w}^{*}\left(\frac{t}{2}\right)+C \int_{t}^{\infty}(M f)_{w}^{*}(s) \frac{d s}{s}
$$

completes the proof of Theorem 1 .

Proof of Theorem 2. Let $\gamma=1 / 2$ in Lemma 3.1. Then for $t>0$ and $n=$ $1,2, \ldots$, iterating inequality $(1.1)$ yields

$$
(T f)_{w}^{*}\left(2^{-n} t\right) \leqslant C \sum_{k=1}^{n}(M f)_{w}^{*}\left(2^{-k-1} t\right)+(T f)_{w}^{*}(t) .
$$

Therefore, by changing the order of summation,

$$
\begin{aligned}
(T f)_{w}^{* *}(t) & =\frac{1}{t} \int_{0}^{t}(T f)_{w}^{*}(s) d s \leqslant \sum_{n=1}^{a}(T f)_{w}^{*}\left(2^{-n} t\right) 2^{-n} \\
& \leqslant \sum_{n=1}^{\infty} 2^{-n}\left(C \sum_{k=1}^{n}(M f)_{w}^{*}\left(2^{-k-1} t\right)+(T f)_{w}^{*}(t)\right) \\
& =(T f)_{w}^{*}(t)+C \sum_{k=1}^{\infty}(M f)_{w}^{*}\left(2^{-k-1} t\right)\left(\sum_{n=k}^{\infty} 2^{-n}\right) \\
& =(T f)_{w}^{*}(t)+4 C \sum_{k=1}^{\infty}(M f)_{w}^{*}\left(2^{-k-1} t\right) 2^{-k-1} \\
& \leqslant \frac{C}{t} \int_{0}^{t}(M f)_{w}^{*}(s) d s+(T f)_{w}^{*}(t) \leqslant C(M f)_{w}^{* *}(t)+(T f)_{w}^{*}(t) .
\end{aligned}
$$

4. Applications. We now consider applications of the results of the last section. In the following four corollaries, we assume that the kernel of the operator $T f$ satisfies the conditions (2.1).

Corollary 4.1. Let $w \in A_{\infty}$. If $(M f)_{w}^{*}(t)$ is finite-valued and $T f$ is bounded except on a set of finite $w$-measure, then $T f$ is finite almost everywhere.

Proof. Choose $t$ so large that $(T f)_{w}^{*}(2 t)<\infty$. By iterating the conclusion of Lemma 3.1, it follows that $(T f)_{w}^{*}(t)<\infty$ for all $t>0$. 
Corollary 4.2. If $w \in A_{\infty}$, then there is a constant $C>0$, independent of $f$ and $p$, such that

$$
\left(\int_{\mathbf{R}^{n}}|T f(x)|^{p} w(x) d x\right)^{1 / p} \leqslant C p\left(\int_{\mathbf{R}^{n}}|M f(x)|^{p}(x) w(x) d x\right)^{1 / p}
$$

for $1 \leqslant p<\infty$.

Proof. Rewrite the conclusion of Theorem 1 as

$$
(T f)_{w}^{*}(t) \leqslant C(M f)_{w}^{*}\left(\frac{t}{2}\right)+C \int_{t}^{\infty}(M f)_{w}^{*}(s) \frac{d s}{s} \leqslant C \int_{t / 4}^{\infty}(M f)_{w}^{*}(s) \frac{d s}{s} .
$$

The conclusion then follows by Hardy's inequality (see [11, p. 196]).

A version of Corollary 4.2 also follows from the "good $\lambda$ " inequality. However, the analogous bound grows exponentially in $p$ rather than linearly. Note also that our proof extends immediately to the Lorentz spaces $L(p, q)$ with no change in the bound.

The next result concerns the space weak $-L^{\infty}$ introduced by Bennett, De Vore and Sharpley [2]. This space consists of the $w$-measurable functions, $f$, such that $f_{w}^{*}(t)$ is finite for $t>0$ and $f_{w}^{* *}(t)-f_{w}^{*}(t)$ is a bounded function of $t$. The "norm" of weak- $L^{\infty}$ is defined by $\|f\|_{\text {weak- } L^{\infty}}=\sup _{t>0}\left(f_{w}^{* *}(t)-f_{w}^{*}(t)\right)$.

Corollary 4.3. Let $w \in A_{\infty}$ and suppose that $T f$ is bounded except on a set of finite $w$-measure. If $f \in L^{\infty}$, then $T f$ is in the space Weak- $L^{\infty}$ and

$$
\|T f\|_{\text {Weak- } L^{\infty}} \leqslant C\|f\|_{\infty} \text {. }
$$

If there is an $\alpha>1$ such that $(M f)_{w}^{* *}(t) \leqslant C[\log (2 / t)]^{\alpha-1}$ for $0<t \leqslant 1$, then there is an $\varepsilon>0$ such that $\exp \left(\varepsilon(T f)^{1 / \alpha}\right)$ is w-integrable over sets of finite w-measure.

Proof. Since $(T f)_{w}^{*}(t)$ is finite for all $t>0$, the first result follows from Theorem 2 , since

$$
(T f)_{w}^{* *}(t)-(T f)_{w}^{*}(t) \leqslant C(M f)_{w}^{* *}(t) \leqslant C\|f\|_{\infty} .
$$

For the second, we use the identity (see [2])

$$
g_{w}^{* *}(t)-g_{w}^{* *}(s)=\int_{t}^{s}\left(g_{w}^{* *}(r)-g_{w}^{*}(r)\right) \frac{d r}{r} .
$$

The assumption on $M f$ implies

$$
\begin{aligned}
(T f)_{w}^{* *}(t)-(T f)_{w}^{* *}(s) & \leqslant C \int_{t}^{s}(M f)_{w}^{* *}(r) d r / r \leqslant C \int_{t}^{s}[\log (2 / r)]^{\alpha-1} d r / r \\
& =C\left([\log (2 / t)]^{\alpha}-[\log (2 / s)]^{\alpha}\right)
\end{aligned}
$$

from which the conclusion follows.

Only the first part of this corollary was known, even in the unweighted case.

We now compare Theorem 1 to previously known rearrangement inequalities, proved under more restrictive hypotheses. To do this, we consider the collection $A_{1}$, an important subclass of $A_{\infty}$. A weight $w \in A_{1}$ if it satisfies the condition

$$
\frac{1}{|Q|} \int_{Q} w(x) d x \leqslant C \underset{x \in Q}{\operatorname{ess} \inf } w(x)
$$

where $C$ is independent of $Q$ (see [8]). 
Since the Hardy-Littlewood maximal function is weak-type $(1,1)$ and strong-type $(\infty, \infty)$ with respect to $A_{1}$ weights, Calderón's theorem [5] implies the following result.

Lemma 4.4. Let $w \in A_{1}$. Then there is a constant $C>0$ so that $(M f)_{w}^{*}(t) \leqslant$ $C f_{w}^{* *}(t)$.

For weights in $A_{1}$ we have

Corollary 4.5. Let $w \in A_{1}$. There exists a constant $C>0$ such that for all $t>0$,

$$
(T f)_{w}^{*}(t) \leqslant C f_{w}^{* *}(t)+C \int_{t}^{\infty} f_{w}^{*}(s) \frac{d s}{s}
$$

and

$$
\begin{aligned}
(T f)_{w}^{* *}(t) & \leqslant C \frac{1}{t} \int_{0}^{t} f_{w}^{* *}(s) d s+C \int_{t}^{\infty} f_{w}^{*}(s) \frac{d s}{s} \\
& \leqslant C \frac{1}{t} \int_{0}^{t} f_{w}^{* *}(s) d s+C \int_{t}^{\infty} f_{w}^{* *}(s) \frac{d s}{s} .
\end{aligned}
$$

The first inequality is proved by Theorem 1 and Lemma 4.4; the second by integrating the first. Only unweighted versions of these inequalities were previously known. Inequality (i) was proved by Bennett and Rudnick [3]; the last inequality was proved by O'Neil and Weiss [9] for the Hilbert transform.

5. Generalizations to Dini kernels. The techniques developed in $\S 3$ extend easily to operators with kernels satisfying conditions weaker than (2.1). In this section we discuss one such class of kernels.

Let $\Sigma=\Sigma_{n-1}=\left\{x \in \mathbf{R}^{n}:|x|=1\right\}$ and, for $x \neq 0, x^{\prime}=x /|x| \in \Sigma$. Suppose that $K(x)$ is a positively homogeneous kernel of degree $-n$, has mean value zero on $\Sigma$, and $K \in L^{r}(\Sigma)$ for some $r, 1<r \leqslant \infty$. We say that $K$ satisfies the $L^{r}$-Dini condition ( $K \in L^{r}$-Dini) if

$$
\int_{0}^{1} \omega_{r}(t) \frac{d t}{t}<\infty
$$

where

$$
\omega_{r}(t)=\sup \left(\int_{\Sigma}\left|K\left(\rho x^{\prime}\right)-K\left(x^{\prime}\right)\right|^{r} d x^{\prime}\right)^{1 / r}
$$

with the sup taken over all rotations, $\rho$, of $\Sigma$ with magnitude $|\rho|=\sup _{x \in \Sigma}|\rho x-x|$ $<t$.

In order to extend our results to Dini kernels, we recall the following lemma from [7].

Lemma 5.1. Let $K \in L^{r}(\Sigma) \cap L^{r}$-Dini. There exists a constant $\alpha>0$ such that if $|y|<\alpha R$, then

$$
\begin{gathered}
\left(\int_{\{x: R<|x|<2 R\}}|K(x-y)-K(x)|^{r} d x\right)^{1 / r} \\
\leqslant C R^{-n+n / r}\left(\frac{|y|}{R}+\int_{|y| / 2 R}^{|y| / R} \omega_{r}(t) \frac{d t}{t}\right) .
\end{gathered}
$$


For $1<r<\infty$, we define the generalized Hardy-Littlewood maximal operator $M_{p} f$ by $M_{p} f(x)=\left(M\left(|f|^{p}\right)(x)\right)^{1 / p}$. The analog of Lemma 3.1 for Dini kernels is

LEMMA 5.2. Let $T f=\sup _{\varepsilon>0}\left|T_{\varepsilon} f\right|$ be a maximal singular integral operator with a kernel $K \in L^{r}(\Sigma) \cap L^{r}$-Dini, where $1<r \leqslant \infty$, and let $w \in A_{\infty}$. For all $\gamma, 0<\gamma<1$, there is a constant $C=C(\gamma)>0$ such that

$$
(T f)_{w}^{*}(t) \leqslant C\left(M_{r^{\prime}} f\right)_{w}^{*}(\gamma t)+(T f)_{w}^{*}(2 t) \quad \text { for all } t>0 .
$$

The proof is the same as the one used to prove Lemma 3.1 with two changes. The first is that we use Lemma 5.1 to fix the ratio $|Q| /\left|Q_{k}\right|$. The other change deals with the estimate of the term

$$
\int_{\left\{y:\left|x_{k}-y\right|>\delta\right\}}\left|K\left(x_{k}-y\right)-K(x-y) \| f(y)\right| d y .
$$

Using the argument from the proof of Theorem 4 of [7], this integral is bounded by a constant times $M_{r^{\prime}} f(x)$.

Under the hypothesis of Lemma 5.2, we obtain exact analogs of Theorems 1 and 2 with $M f$ replaced by $M_{r^{\prime}} f$.

\section{BIBLIOGRAPHY}

1. C. Bennett, R. De Vore and R. Sharpley, Maximal singular integrals on $L^{\infty}$, Colloq. Math. Soc. János Bolyai, no. 35, Functions, Series, Operators, Budapest, North-Holland, Amsterdam, 1980, pp. $233-235$.

2. Weak- $L^{\infty}$ and BMO, Ann. of Math. (2) 113 (1981), 601-611.

3. C. Bennett and K. Rudnick, On Lorentz-Zygmund spaces, Dissertationes Math. 175 (1980), 1-67.

4. C. Bennett and R. Sharpley, Weak-type inequalities for $H^{p}$ and BMO, Proc. Sympos. Pure Math., vol. 35, Part 1, Amer. Math. Soc., Providence, R. I., 1979, pp. 201-229.

5. A. P. Calderón, Spaces between $L^{1}$ and $L^{\infty}$ and the theorem of Marcinkiewicz, Studia Math. 26 (1966), 273-299.

6. R. R. Coifman and C. Fefferman, Weighted norm inequalities for maximal functions and singular integrals, Studia Math. 51 (1974), 241-250.

7. D. S. Kurtz and R. L. Wheeden, Results on weighted norm inequalities for multipliers, Trans. Amer. Math. Soc. 225 (1979), 343-362.

8. B. Muckenhoupt, Weighted inequalities for the Hardy maximal function, Trans. Amer. Math. Soc. 165 (1972), 207-226.

9. R. O'Neil and G. Weiss, The Hilbert transform and rearrangement of functions, Studia Math. 23 (1963), 189-198.

10. E. M. Stein, Singular integrals and differentiability properties of functions, Princeton Univ. Press, Princeton, N. J., 1970.

11. E. M. Stein and G. Weiss, Introduction to Fourier analysis on Euclidean spaces, Princeton Univ. Press, Princeton, N. J., 1971.

Department of Mathematical Sciences, New Mexico State University, las Cruces, New MEXICO 88003 